\title{
CCD PHOTOMETRY OF CANDIDATE RR LYRAE STARS IN THE SLOAN DIGITAL SKY SURVEY
}

\author{
C. Wu, ${ }^{1}$ Y. L. QIU, ${ }^{1}$ J. S. DenG, ${ }^{1}$ J. Y. Hu, ${ }^{1}$ AND Y. H. ZhaO ${ }^{1}$ \\ Received 2004 December 21; accepted 2005 June 27
}

\begin{abstract}
We present unfiltered CCD photometry of a sample of 71 candidate RR Lyrae stars during 2003 and 2004, out of 148 candidate stars that were selected from Sloan Digital Sky Survey (SDSS) commissioning data by Ivezić and coworkers. We obtain light curves of 69 candidate stars and present a catalog of their positions, amplitudes, periods, types, and mean magnitudes. We confirm that the 69 stars are true RR Lyrae variables based on periods and light curves, for the first time for 41 of them. We identify 57 type ab, 11 type c, and 1 type d (double-mode) in our sample. The doublemode RR Lyrae star shows properties similar to globular cluster stars and are unlike field stars of the Galactic halo in the Petersen diagram. We also find one RR Lyrae star that is related to Pal 5 and recover another. In our sample, only two candidate stars do not have a reasonable light curve. We conclude that the ratio of contamination in our sample is less than $\sim 8 \%$, which shows that the criteria used by Ivezić and coworkers to select RR Lyrae stars from the SDSS two-epoch data are reliable.
\end{abstract}

Key words: Galaxy: halo — Galaxy: structure — stars: variables: other

Online material: color figures, machine-readable table

\section{INTRODUCTION}

The origin of the Galactic stellar halo has been a long-standing astronomical problem. It has been debated whether it formed during the rapid collapse of a large gas cloud (Eggen et al. 1962) or through the slow merger of satellite galaxies (Searle 1977; Searle \& Zinn 1978), while several authors have argued for a combination of both processes based on observations of halo stars and globular clusters (Chiba \& Beers 2001; Norris 1994; Zinn 1993; Majewski 1993). On the one hand, there has been strong observational evidence in favor of the merger picture in recent years, including the tidally distorted Sagittarius dwarf galaxy (Ibata et al. 2001; Majewski et al. 2003; Newberg et al. 2002; Yanny et al. $2000,2003)$, the presence of extratidal stars around many dwarf spherodial satellites (Gould et al. 1992; Irwin \& Hatzidimitriou 1995; Kuhn et al. 1996; Majewski et al. 2000), and the recognition that the halo and bulge are distinct components with different formation mechanisms (see Wyse 1999a, 1999b for a review). On the other hand, theoretical models of the growth of galaxies through cold dark matter (Bullock et al. 2000 and references therein) have made great progress. According to these models, galaxies similar to the Milky Way formed through the merger of a large number of dark matter subhalos. The stars that formed in these subhalos, which resembled dwarf galaxies, make up today's stellar halo. This picture appears to be in good agreement with the predictions for the RR Lyrae star (RRLS) distribution in the outer stellar halo (Bullock et al. 2001).

RRLSs are especially useful probes of the stellar halo (Bullock et al. 2001) because they are relatively easy to identify, luminous enough to be detected out to large distances $(r \sim 100 \mathrm{kpc}$, corresponding to $r^{\prime} \sim 21 \mathrm{mag}$ ), and, as nearly standard candles, able to yield three-dimensional maps (see Kinman 2002 and references therein). Since Baade (1944), RRLSs have also been adopted to trace the old stellar component in the Galaxy (see Bono 2003 and references therein). The use of RRLSs as stellar tracers received a new spin during the last few years. The photometric Sloan Dig-

\footnotetext{
${ }^{1}$ National Astronomical Observatories, Chinese Academy of Sciences, Beijing 100012,China; wuchao@lamost.org, qiuyl@bao.ac.cn, jsdeng@bao.ac.cn, hjy@ bao.ac.cn, yzhao@lamost.org.
}

ital Sky Survey (SDSS; Ivezić et al. 2000) and QUEST survey (Vivas et al. 2001; Vivas \& Zinn 2003; Zinn et al. 2004) identified a local overdensity of RRLSs in the Galactic halo. Their argument that one of the clumps originated from the Sagittarius dwarf galaxy was recently verified by spectroscopic data of Vivas et al. (2005), although these observations suggested that some revisions are needed for models of the disruption of Sagittarius.

The SDSS sample of Ivezić et al. (2000) comprises 148 candidate RRLSs that they selected from $\sim 930,000$ stars and covers $\sim 100 \mathrm{deg}^{2}$ of sky, based on two-epoch photometry and using variability and colors as selection criteria. Their analysis of the spatial distribution of RRLSs in the Galactic halo was based on the assumption that their candidates are real RRLSs. To confirm their results, detailed photometry of these candidates is needed that can be used to determine their periods and light curves. Furthermore, their RRLS selection criteria based on two-epoch photometry, if verified, could be very useful in extending the RRLS sample in the Galactic halo and hence deserve verification.

In this paper we present the periods and light curves of 69 RRLSs out of the SDSS candidate sample (Ivezić et al. 2000). The details of our observations and data reduction are presented in $\S 2$. Our method of period finding is explained in $\S 3$. In $\S 4$ we present a catalog of the 69 RRLSs and discuss their light curves. We also analyze the spatial distribution of RRLSs in the halo in this section and discuss the RRLSs in Pal 5. Our results are summarized in $\S 5$.

\section{OBSERVATION AND DATA REDUCTION}

Our CCD images were obtained without a filter using two telescope systems, the 0.6 and $0.8 \mathrm{~m}$ telescopes at the Xinglong station of the National Astronomical Observatory of China (NAOC) during 2003 and 2004. The $0.6 \mathrm{~m}$ telescope was equipped with a $1340 \times 1340$ pixel CCD at the Cassegrain focus of focal ratio $\mathrm{f} / 15$. The detector has a pixel size of $20 \mu \mathrm{m}$, producing a scale on the chip of $0^{\prime \prime} 46$ pixel $^{-1}$ and a $10^{\prime} \times 10^{\prime}$ field of view. Its dark current is very low because of its cooling by liquid nitrogen. The $0.8 \mathrm{~m}$ telescope (TNT), which is jointly administrated by Tsinghua University and the NAOC, has a CCD camera mounted at the Cassegrain focus of $\mathrm{f} / 10$, with $1024 \times 1024$ pixels. The pixel size 
is $13 \mu \mathrm{m}$, yielding a scale of about 0.34 pixel $^{-1}$ with a $6^{\prime} \times 6^{\prime}$ field of view. The camera is thermoelectrically cooled to about $-50^{\circ} \mathrm{C}$, so the dark current is relatively high.

Our observational strategy was to obtain extensive photometric coverage of a variable to get good light curves. Typically, 40 observations are enough to obtain good phase interval coverage where the light curve is changing rapidly (see, e.g., Layden 1997). However, in practice, we obtained as many as $\sim 50$ observations for one star on average (see Table 1). Each CCD image taken by the $0.6 \mathrm{~m}$ telescope had $60 \mathrm{~s}$ exposures, while the exposures from the $0.8 \mathrm{~m}$ depended on the magnitudes and weather. The average exposure was $\sim 240 \mathrm{~s}$ for a star with magnitude $r^{\prime}=18$. The time spacing of our images was frequently determined by clouds and ranged from a few hours, to days, weeks, and months. This irregular spacing of the observations was helpful in removing some of the aliasing in our period search. We collected $\sim 4000$ image frames during 2003 and 2004. Most observations were collected during the months of February and May of each year, which is partly a consequence of the weather patterns at the Xinglong observatory.

Preliminary reductions, including bias subtractions and dark and flat-field corrections, were performed with standard IRAF ${ }^{2}$ packages for all the data. The mean value of seeing was $\sim 2$ ". 1 ; thus, we eliminated some bad-quality images, e.g., with FWHM $>4$."0.

Aperture photometry was performed on all the objects. We chose the images of best quality in each field as templates. First, for one field we performed the photometry of the template. According to the results, we excluded from being comparison stars those that were very close to any bright stars or were contaminated by close neighbors in crowded regions. Then we marked the program object and comparison stars in the template. Finally, the automatic processing program (Kong et al. 2002), integrated scripts that ran the IRAF packages, produced the results of the photometry for all marked stars. In this process, photometry was done with the APPHOT package in IRAF, and the aperture was set to $1.5 \times$ FWHM considering the magnitudes of the objects in order to reduce errors in the faintest stars, where the FWHM was the average for all chosen stars in each image. A whole set of properties (magnitudes, errors, FWHM, position, shape, parameters, etc.) of photometry were provided in the results, which helped to eliminate bad measurements.

We plotted CCD photometric errors against the $r^{\prime}$ magnitudes of our photometric results in Figure $1 a$. Each point represents the mean CCD photometric error from the various images for one star. The fitted lines indicate the typical errors of our data measured by the 0.6 and $0.8 \mathrm{~m}$ telescopes.

The comparison stars in each field were chosen manually. Stars that were too bright saturated the CCD pixels on very clear nights, while ones that were too faint were not well exposed and had low signal-to-noise ratios (S/N). Typically, our selected comparison stars were of 12-16 mag, with approximately five for each field. Usually we calculated the differential magnitude from the average difference between the variable and all constant comparison stars in the same field.

In order to verify the constancy of the comparison stars, we measured the differential magnitudes between each pair of comparison stars and calculated the rms of the sequence of measurements. The rms values of all pairs of comparison stars are statistically illustrated in Figure $1 b$. A Gaussian fitting to these values shows that the most probable rms value is $\sim 0.03 \mathrm{mag}$, which is $\sim 0.02$ mag larger than the photometric error of a typical com-

\footnotetext{
${ }^{2}$ IRAF is written and distributed by the National Optical Astronomy Observatory.
}

parison star. This deviation probably resulted from the color terms that were not taken into consideration in the differential photometry. In this work, we only selected comparison stars that had an rms value less than $\sim 0.04 \mathrm{mag}$.

We used unfiltered CCDs in order to increase the efficiency of our measurements. This enabled us to save a lot of telescope time and obtain more extensive photometric phase coverage to improve the photometric time-series precision. In order to determine the distance, unfiltered CCD magnitudes were transformed to standard SDSS $r^{\prime}$ magnitudes (Smith et al. 2002) using an empirical method based on the CCD sensitivity function. A similar method was used by Riess et al. (1999) to transform supernova magnitudes observed with nonstandard filters to a standard system. We summarize our method as follows.

For each field, corresponding to one program object, we first picked out one image of high $\mathrm{S} / \mathrm{N}$ and measured the differences in the unfiltered magnitude between all local standard stars and one randomly marked comparison star. Referring to an individual unfiltered CCD system as a nonstandard passband system " $W$," we express the magnitude difference as

$$
\Delta W=W_{\mathrm{ls}}-W_{\text {com }} .
$$

At the same time, we can also get the differences in the $r^{\prime}$ magnitude between the local standard stars and the comparison star, i.e.,

$$
\Delta r^{\prime}=r_{\mathrm{ls}}^{\prime}-r_{\mathrm{com}}^{\prime}
$$

where the $r^{\prime}$ magnitudes of the local standard stars refer to the SDSS Second Data Release. The relationship between $\Delta r^{\prime}$ and $\Delta W$ for any specific field can be well fitted using an empirical linear function,

$$
\Delta r^{\prime}=C_{0}+C_{v w} \Delta W
$$

where the fitting parameters have small dispersions. This procedure was employed for all fields. The results for a typical field are shown in Figure 2. Using equation (3), the $r^{\prime}$ magnitude of the program object can be easily obtained. The advantage of this method is that it does not require explicit knowledge of the unfiltered response function.

The mean uncertainty in the $r^{\prime}$ magnitude introduced by this transformation is $\sim 0.05 \mathrm{mag}$, which for our data gives a valuable precision of $\sim 5 \%$. For unfiltered images with $\mathrm{S} / \mathrm{N} \geq 20$, the dominant source of uncertainty in the $r^{\prime}$ magnitude comes from the pseudocolor term, $C_{v w}$, in equation (3). For observations with $\mathrm{S} / \mathrm{N} \leq 10$, however, the uncertainty is dominated by the statistical uncertainty of the measured flux of the variables.

The total errors in the $r^{\prime}$ magnitudes of the 69 RRLSs are listed in column (5) of Table 1 and are about $\sim 0.07$ mag on average. These were computed by adding in quadrature the errors in the aperture magnitudes of the variable, mean standard errors introduced by comparison stars, and magnitude transformation errors.

\section{PERIOD FINDING}

We used the phase dispersion minimization (PDM) method, proposed by Lafler \& Kinman (1965) and developed by Stellingwerf (1978), to find periods for the RRLSs. This method has some advantages over other period-searching methods. It is most suitable for the case in which only a few observations over a limited period of time are available. Compared with Fourier techniques, it can obtain accurate periods and nonsinusoidal light curves for the component oscillations (Stellingwerf 1978). It 
TABLE 1

RR Lyrae Stars

\begin{tabular}{|c|c|c|c|c|c|c|c|c|c|c|}
\hline $\begin{array}{l}\text { ID } \\
(1)\end{array}$ & $\begin{array}{c}\alpha(\mathrm{J} 2000.0) \\
(2)\end{array}$ & $\begin{array}{c}\delta(\mathrm{J} 2000.0) \\
(3)\end{array}$ & $\begin{array}{l}N \\
(4)\end{array}$ & $\begin{array}{l}\text { Amplitude } \\
\text { (5) }\end{array}$ & $\begin{array}{c}\text { Period } \\
\text { (days) } \\
(6)\end{array}$ & $\begin{array}{c}E_{\max } \\
(\mathrm{HJD}) \\
(7)\end{array}$ & $\begin{array}{l}\text { Type } \\
(8)\end{array}$ & $\begin{array}{l}r^{\prime} \\
(9)\end{array}$ & $\begin{array}{l}\sigma_{r^{\prime}} \\
(10)\end{array}$ & $\begin{array}{l}\text { Tag } \\
\text { (11) }\end{array}$ \\
\hline $1 \ldots \ldots \ldots \ldots \ldots$ & 104902.61 & +010500.6 & 48 & 0.92 & 0.552281 & 2452733.0183 & $a b$ & 17.35 & 0.07 & \\
\hline $2 \ldots \ldots \ldots \ldots$ & 105314.69 & +01 1201.4 & 55 & 0.44 & 0.322484 & 2452733.1574 & $\mathrm{c}$ & 14.75 & 0.06 & $\ldots$ \\
\hline 3................ & 105926.11 & -005927.6 & 47 & 0.60 & 0.450446 & 2452732.9266 & $a b$ & 18.07 & 0.09 & $\mathrm{q}$ \\
\hline $5 \ldots \ldots \ldots \ldots$ & 110838.26 & $\begin{array}{lll}-00 & 05 & 14.3\end{array}$ & 60 & 0.42 & 0.615099 & 2452733.3179 & $a b$ & 15.07 & 0.06 & $\mathrm{q}$ \\
\hline $6 \ldots \ldots \ldots \ldots . . .$. & $11 \quad 1010.79$ & +010732.9 & 54 & 0.50 & 0.378346 & 2453047.1864 & $\mathrm{c}$ & 17.26 & 0.06 & $\ldots$ \\
\hline $7 \ldots \ldots \ldots \ldots$ & 111705.98 & -003424.0 & 44 & 0.87 & 0.588000 & 2452732.7823 & $a b$ & 17.45 & 0.09 & $\ldots$ \\
\hline $8 \ldots \ldots \ldots \ldots$ & 112425.37 & -000919.7 & 27 & 1.03 & 0.691869 & 2452754.5840 & $a b$ & 17.70 & 0.09 & $\mathrm{q}$ \\
\hline $9 \ldots \ldots \ldots \ldots . .$. & 112837.73 & -000112.6 & 52 & 1.07 & 0.565301 & 2453046.7289 & $a b$ & 18.58 & 0.07 & $\mathrm{q}$ \\
\hline $11 \ldots \ldots \ldots . . .$. & 113814.16 & +010528.2 & 48 & 0.86 & 0.636825 & 2453047.5456 & $a b$ & 18.87 & 0.08 & $\ldots$ \\
\hline $12 \ldots \ldots \ldots \ldots$ & 114542.24 & +00 2314.6 & 43 & 0.65 & 0.587633 & 2452733.0016 & $a b$ & 17.83 & 0.08 & $\ldots$ \\
\hline $14 \ldots \ldots \ldots \ldots$ & 115113.99 & +004505.7 & 58 & 0.62 & 0.608022 & 2452733.3820 & $a b$ & 15.77 & 0.06 & $\ldots$ \\
\hline $15 \ldots \ldots \ldots \ldots$ & 115534.40 & -003601.9 & 43 & 0.29 & 0.596184 & 2452733.0141 & $a b$ & 16.94 & 0.06 & $\mathrm{q}$ \\
\hline $16 \ldots \ldots \ldots \ldots$ & 115628.60 & +011223.9 & 42 & 0.70 & 0.452015 & 2452733.0220 & $a b$ & 17.46 & 0.07 & $\ldots$ \\
\hline $17 \ldots \ldots \ldots \ldots$ & 115706.95 & -005507.9 & 56 & 0.84 & 0.552716 & 2453053.8103 & $a b$ & 17.62 & 0.06 & $\mathrm{q}$ \\
\hline $18 \ldots \ldots \ldots \ldots$ & 115724.21 & -005358.2 & 43 & 0.90 & 0.554266 & 2453061.9848 & $a b$ & 18.22 & 0.07 & $\mathrm{q}$ \\
\hline $19 \ldots \ldots \ldots \ldots$ & 120047.92 & +00 4611.1 & 57 & 0.46 & 0.343466 & 2452698.8781 & $\mathrm{c}$ & 17.03 & 0.08 & $\ldots$ \\
\hline $20 \ldots \ldots \ldots \ldots$ & 120730.94 & -000412.6 & 58 & 0.54 & 0.543244 & 2452699.1234 & $a b$ & 17.42 & 0.07 & $\mathrm{q}$ \\
\hline $21 \ldots \ldots \ldots \ldots$ & 121329.64 & -010151.9 & 38 & 0.72 & 0.612120 & 2453053.8683 & $a b$ & 17.18 & 0.07 & $\ldots$ \\
\hline $22 \ldots \ldots \ldots \ldots$ & 121507.76 & +004930.1 & 45 & 0.70 & 0.354983 & 2453054.2795 & $\mathrm{c}$ & 17.78 & 0.07 & $\ldots$ \\
\hline $23 \ldots \ldots \ldots$ & 121527.79 & -005256.5 & 59 & 0.74 & 0.523110 & 2452733.3378 & $a b$ & 16.05 & 0.06 & $\mathrm{q}$ \\
\hline $24 \ldots \ldots \ldots \ldots$ & 121803.72 & +001448.9 & 33 & 1.04 & 0.596015 & 2453058.0100 & $a b$ & 18.03 & 0.07 & $\ldots$ \\
\hline $25 \ldots \ldots \ldots \ldots$ & $1222 \quad 28.39$ & $\begin{array}{lll}-01 & 02 & 16.3\end{array}$ & 64 & 0.48 & 0.800898 & 2452699.2477 & $a b$ & 14.60 & 0.07 & $\ldots$ \\
\hline $26 \ldots \ldots \ldots \ldots$ & 122501.92 & +01 1407.9 & 56 & 0.61 & 0.728355 & 2452699.2979 & $a b$ & 16.14 & 0.06 & $\ldots$ \\
\hline $27 \ldots \ldots \ldots \ldots$ & 122529.03 & +011420.8 & 62 & 0.75 & 0.596109 & 2452698.5423 & $a b$ & 15.71 & 0.06 & $\ldots$ \\
\hline $29 \ldots \ldots \ldots . . .$. & 124032.87 & $\begin{array}{lll}-00 & 03 & 12.9\end{array}$ & 47 & 0.42 & 0.164130 & 2452699.1646 & $\mathrm{c}$ & 17.15 & 0.07 & $\mathrm{q}$ \\
\hline $30 \ldots \ldots \ldots \ldots$ & 124046.56 & +005006.2 & 30 & 0.60 & 0.319512 & 2453062.1433 & $\mathrm{c}$ & 17.97 & 0.12 & $\ldots$ \\
\hline $31 \ldots \ldots \ldots \ldots$ & 124136.64 & +011306.5 & 59 & 0.70 & 0.605124 & 2453062.4251 & $\mathrm{ab}$ & 17.07 & 0.08 & $\ldots$ \\
\hline $32 \ldots \ldots \ldots \ldots$ & 124224.91 & -001203.1 & 56 & 0.38 & 0.360981 & 2452699.0510 & $\mathrm{c}$ & 14.63 & 0.08 & $\mathrm{q}$ \\
\hline $34 \ldots \ldots \ldots \ldots$ & 125208.73 & -002931.8 & 69 & 0.87 & 0.562213 & 2452698.7822 & $a b$ & 15.52 & 0.06 & $\mathrm{q}$ \\
\hline 37............. & $13 \quad 11 \quad 17.74$ & -003429.9 & 71 & 1.06 & 0.598353 & 2452699.4648 & $a b$ & 15.60 & 0.06 & $\ldots$ \\
\hline $39 \ldots \ldots \ldots$ & 131757.46 & -000818.8 & 56 & 0.92 & 0.523437 & 2452766.7921 & $a b$ & 17.14 & 0.07 & $\mathrm{q}$ \\
\hline $40 \ldots \ldots \ldots . . .$. & 131806.63 & -003300.2 & 64 & 0.51 & 0.344164 & 2452750.2848 & $\mathrm{c}$ & 15.68 & 0.06 & $\ldots$ \\
\hline $43 \ldots \ldots \ldots \ldots$ & 132624.94 & -002612.1 & 44 & 0.91 & 0.505841 & 2452750.0187 & $a b$ & 16.53 & 0.07 & $\mathrm{q}$ \\
\hline $44 \ldots \ldots \ldots \ldots$ & 132635.08 & +002034.8 & 66 & 1.42 & 0.528236 & 2453138.0244 & $a b$ & 17.69 & 0.06 & $\ldots$ \\
\hline $45 \ldots \ldots \ldots \ldots$ & 132700.05 & -005456.7 & 51 & 0.86 & 0.561698 & 2452750.4104 & $a b$ & 15.00 & 0.06 & $\mathrm{q}$ \\
\hline 47............. & 133252.91 & +004622.6 & 53 & 0.68 & 0.593984 & 2452750.0236 & $a b$ & 16.17 & 0.06 & $\ldots$ \\
\hline $48 \ldots \ldots \ldots . . .$. & 133323.47 & -001159.5 & 50 & 0.93 & 0.596771 & 2453138.4403 & $a b$ & 17.35 & 0.06 & $\mathrm{q}$ \\
\hline $49 \ldots \ldots \ldots . .$. & 133552.24 & -003706.8 & 52 & 0.81 & 0.547890 & 2452750.0374 & $a b$ & 15.10 & 0.06 & $\mathrm{q}$ \\
\hline $53 \ldots \ldots \ldots \ldots$ & $1345 \quad 13.92$ & +002240.0 & 44 & 0.71 & 0.418388 & 2452749.8389 & $\mathrm{c}$ & 17.20 & 0.08 & $\ldots$ \\
\hline $54 \ldots \ldots \ldots \ldots$ & 134521.33 & -000147.4 & 52 & 0.50 & 0.546511 & 2452767.0839 & $a b$ & 14.31 & 0.06 & $\mathrm{q}$ \\
\hline $59 \ldots \ldots \ldots . . . . .$. & 135009.13 & -003414.3 & 58 & 0.62 & 0.522958 & 2452749.7874 & $a b$ & 15.97 & 0.07 & $\ldots$ \\
\hline $61 \ldots \ldots \ldots \ldots$ & 135231.76 & +004350.9 & 56 & 0.93 & 0.537531 & 2452749.8332 & $\mathrm{ab}$ & 17.67 & 0.11 & $\ldots$ \\
\hline $71 \ldots \ldots \ldots \ldots$ & 140606.77 & -003356.8 & 56 & 0.77 & 0.460702 & 2452749.8684 & $a b$ & 15.54 & 0.06 & $\ldots$ \\
\hline $76 \ldots \ldots \ldots$ & 141142.14 & +002248.5 & 57 & 0.82 & 0.577865 & 2452749.7685 & $\mathrm{ab}$ & 15.93 & 0.09 & $\ldots$ \\
\hline 77............... & 141238.55 & -005350.7 & 68 & 0.49 & 0.186607 & 2453138.0175 & $\mathrm{c}$ & 15.20 & 0.07 & $\mathrm{q}$ \\
\hline $79 \ldots \ldots \ldots \ldots$ & 141543.43 & -000613.0 & 56 & 0.83 & 0.572610 & 2452750.1031 & $a b$ & 17.30 & 0.08 & $\mathrm{q}$ \\
\hline $82 \ldots \ldots \ldots \ldots$ & 141807.36 & +002302.6 & 52 & 0.41 & 0.618395 & 2452749.8027 & $a b$ & 15.06 & 0.06 & $\ldots$ \\
\hline $91 \ldots \ldots \ldots \ldots$ & 142356.74 & -003428.5 & 55 & $0.46,0.25$ & $0.354260,0.474188$ & 2452749.9385 & $\mathrm{~d}$ & 15.93 & 0.07 & $\ldots$ \\
\hline $97 \ldots \ldots \ldots . . . .$. & 142808.95 & -001148.3 & 49 & 0.63 & 0.547472 & 2452749.8151 & $a b$ & 16.40 & 0.07 & $\mathrm{q}$ \\
\hline $103 \ldots \ldots \ldots$ & 143614.78 & +010825.9 & 91 & 1.04 & 0.483590 & 2452749.8151 & $a b$ & 15.12 & 0.06 & $\ldots$ \\
\hline $104 \ldots \ldots \ldots$ & 143713.36 & +00 1623.0 & 90 & 0.67 & 0.602988 & 2452750.1715 & $a b$ & 15.89 & 0.06 & $\ldots$ \\
\hline $109 \ldots \ldots \ldots$ & 144618.52 & +00 1321.2 & 54 & 0.59 & 0.730896 & 2452768.6455 & $\mathrm{ab}$ & 15.41 & 0.07 & $\ldots$ \\
\hline $110 \ldots \ldots \ldots$ & 144720.41 & -000101.7 & 47 & 0.76 & 0.734595 & 2453138.2790 & $\mathrm{ab}$ & 17.39 & 0.06 & $\mathrm{q}$ \\
\hline $111 \ldots \ldots \ldots$ & 144939.60 & -002943.9 & 53 & 1.03 & 0.735997 & 2452769.5135 & $\mathrm{ab}$ & 17.22 & 0.10 & $\mathrm{q}$ \\
\hline $115 \ldots \ldots \ldots$ & 145719.71 & -005328.0 & 65 & 0.77 & 0.554167 & 2452769.2273 & $a b$ & 15.58 & 0.06 & $\mathrm{q}$ \\
\hline $120 \ldots \ldots \ldots$ & 150337.35 & $\begin{array}{lll}-00 & 28 & 12.8\end{array}$ & 56 & 0.56 & 0.612234 & 2452753.7732 & $a b$ & 14.89 & 0.06 & $\ldots$ \\
\hline $121 \ldots \ldots \ldots$ & 150545.38 & -000505.3 & 65 & 0.71 & 0.426879 & 2452769.1771 & $a b$ & 16.63 & 0.07 & $\ldots$ \\
\hline $124 \ldots \ldots \ldots$ & 150916.76 & +001947.2 & 64 & 0.57 & 0.561183 & 2452769.0704 & $a b$ & 16.30 & 0.07 & $\ldots$ \\
\hline $128 \ldots \ldots \ldots$ & 151435.44 & -002959.7 & 64 & 0.70 & 0.621765 & 2452768.9872 & $a b$ & 15.60 & 0.09 & $\ldots$ \\
\hline $130 \ldots \ldots \ldots$ & 151557.21 & -000653.2 & 50 & 0.58 & 0.293345 & 2453138.3482 & $\mathrm{c}$ & 17.43 & 0.06 & $\mathrm{q}$ \\
\hline $133 \ldots \ldots \ldots . .$. & 151823.60 & +002122.2 & 84 & 1.14 & 0.529198 & 2453156.2681 & $a b$ & 17.36 & 0.06 & $\ldots$ \\
\hline $134 \ldots \ldots \ldots \ldots$ & 152014.18 & -002603.0 & 66 & 0.98 & 0.643060 & 2452769.6688 & $a b$ & 15.01 & 0.07 & $\mathrm{q}$ \\
\hline $135 \ldots \ldots \ldots$ & 152122.93 & -000530.9 & 61 & 1.08 & 0.540194 & 2452769.2806 & $a b$ & 17.43 & 0.10 & $\mathrm{q}$ \\
\hline
\end{tabular}


TABLE 1 - Continued

\begin{tabular}{|c|c|c|c|c|c|c|c|c|c|c|}
\hline $\begin{array}{l}\text { ID } \\
(1)\end{array}$ & $\begin{array}{c}\alpha(\mathrm{J} 2000.0) \\
\quad(2)\end{array}$ & $\begin{array}{c}\delta(\mathrm{J} 2000.0) \\
(3)\end{array}$ & $\begin{array}{l}N \\
(4)\end{array}$ & $\begin{array}{l}\text { Amplitude } \\
\text { (5) }\end{array}$ & $\begin{array}{c}\text { Period } \\
\text { (days) } \\
(6)\end{array}$ & $\begin{array}{c}E_{\max } \\
(\mathrm{HJD}) \\
(7)\end{array}$ & $\begin{array}{l}\text { Type } \\
(8)\end{array}$ & $\begin{array}{l}r^{\prime} \\
(9)\end{array}$ & $\begin{array}{c}\sigma_{r^{\prime}} \\
(10)\end{array}$ & $\begin{array}{l}\text { Tag } \\
(11)\end{array}$ \\
\hline $137 \ldots \ldots \ldots \ldots \ldots \ldots$ & 152318.61 & -005520.9 & 58 & 0.74 & 0.609840 & 2452734.3149 & $\mathrm{ab}$ & 16.73 & 0.07 & $\mathrm{q}$ \\
\hline 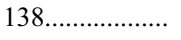 & 152547.10 & +00 2409.5 & 44 & 1.16 & 0.352659 & 2453155.9224 & $\mathrm{ab}$ & 17.58 & 0.11 & $\ldots$ \\
\hline $144 \ldots \ldots \ldots \ldots \ldots \ldots$ & 153443.29 & -002937.9 & 63 & 0.81 & 0.580523 & 2452769.4591 & $a b$ & 15.82 & 0.06 & $\ldots$ \\
\hline $145 \ldots \ldots \ldots \ldots \ldots$ & 153502.96 & +00 1421.5 & 62 & 0.51 & 0.534233 & 2452734.2866 & $a b$ & 15.77 & 0.08 & $\ldots$ \\
\hline 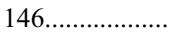 & 153518.04 & +00 1405.9 & 67 & 0.64 & 0.811415 & 2453148.8009 & $\mathrm{ab}$ & 17.29 & 0.07 & $\ldots$ \\
\hline 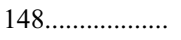 & 153938.01 & +011124.2 & 55 & 0.70 & 0.708399 & 2452768.8132 & $a b$ & 17.05 & 0.08 & $\ldots$ \\
\hline
\end{tabular}

Notes.-Units of right ascension are hours, minutes, and seconds, and units of declination are degrees, arcminutes, and arcseconds. A "q" in the last column indicates that the star is identified by Vivas et al. (2004). Table 1 is also available in machine-readable form in the electronic edition of the Astronomical Journal.

works particularly well on discrete observations and hence allows for completely automatic period searching.

We summarize the improved PDM of Stellingwerf(1978) and the relevant definitions as follows: Given the $i$ th observation $\left(m_{i}\right.$, $t_{i}$ ), the variance of $m$ is

$$
\sigma^{2}=\frac{\sum\left(m_{i}-\bar{m}\right)^{2}}{N-1}
$$

where $N$ is the number of observational points and $\bar{m}=$ $\sum m_{i} / N$ is the mean magnitude. Meanwhile, for any subset of $m_{i}$, the subsample variance $s_{j}^{2}$ is defined exactly as in equation (4). Suppose $M$ distinct subsamples are chosen from the sample and they have variances $s_{j}(j=1, M)$ and contain $n_{j}$ data points. The overall variance for all samples is then given by

$$
s^{2}=\frac{\sum\left(n_{j}-1\right) s_{j}^{2}}{\sum n_{j}-M}
$$

as a consequence of equation (4).

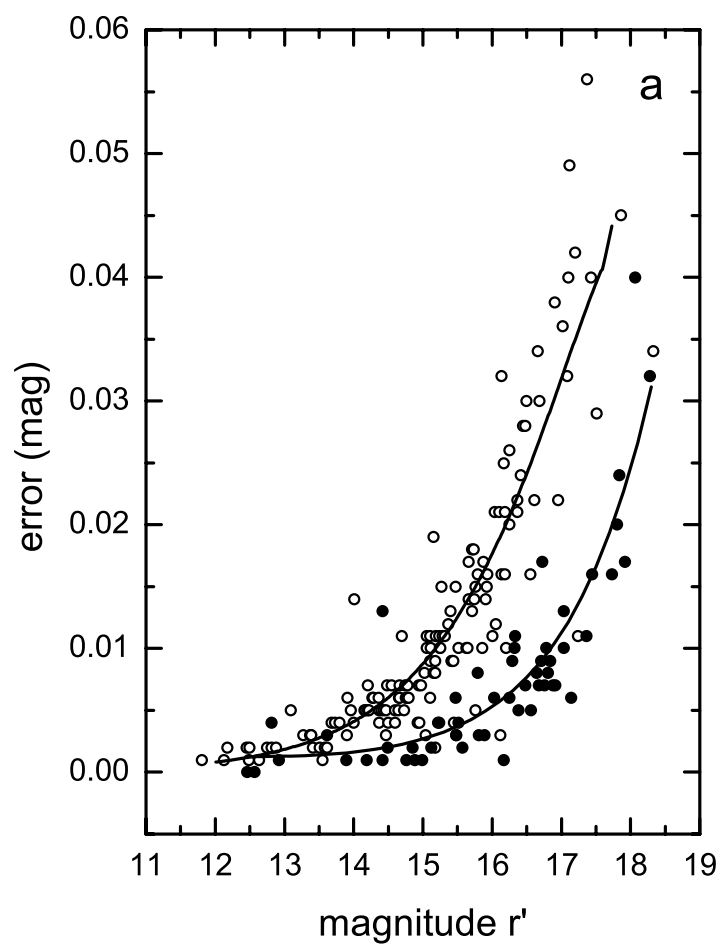

Given a trial period $\Pi$, we can get a phase vector $\phi_{i}=t_{i} / \Pi-$ $\left[t_{i} / \Pi\right]$. Then we pick $M$ subsamples from $m$ using the criterion that all the members of subsample $j$ have similar $\phi_{i}$. Usually the full phase interval $(0,1)$ is divided into fixed bins, but the subsamples can be chosen in any way that satisfies the criterion. The overall variance of these subsamples gives a measure of the scatter around the mean light curve defined by the means of $m_{i}$ in each subsample. The statistic can be defined by

$$
\Theta=s^{2} / \sigma^{2}
$$

where $s^{2}$ is given by equation (5) and $\sigma^{2}$ is given by equation (4). If $\Pi$ is not the true period, then $s^{2} \approx \sigma^{2}$ and $\Theta \approx 1$, whereas if $\Pi$ is the correct period, $\Theta$ will reach a local minimum compared with the neighboring periods, hopefully near zero. When we set a trial period ranging from 0.1 to 2 days for each star, the period corresponding to the minimum value of $\Theta$ is the best one in the trial range. With the best period, we can plot a light curve that will look as expected for this type of variable. But for aperiodic variables

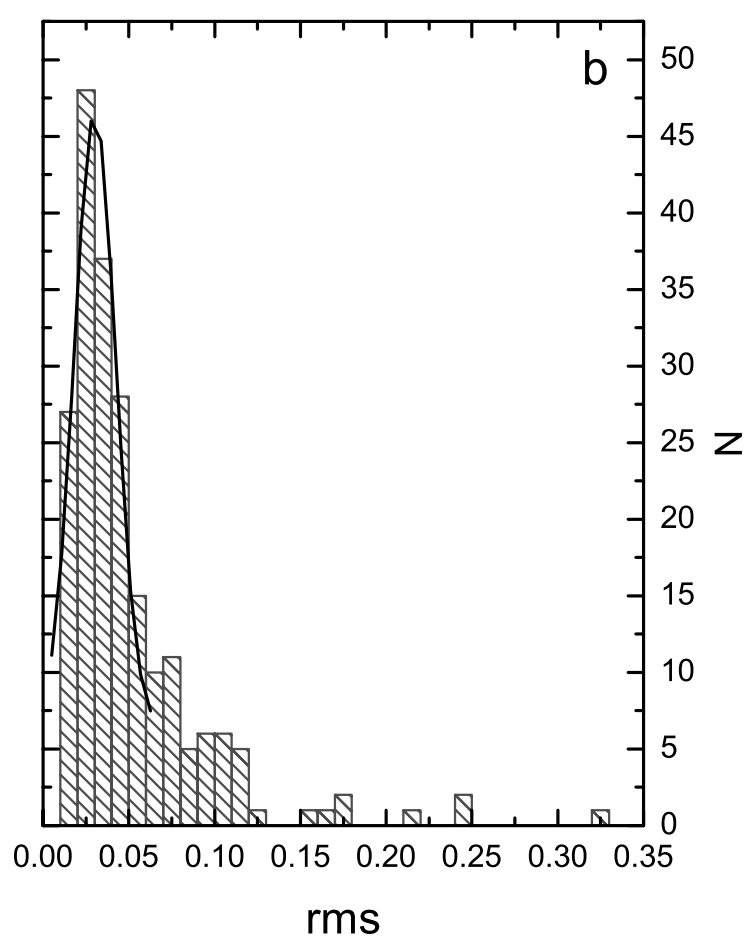

FIG. 1.-(a) CCD photometric errors of our data. The open and filled circles show data measured by the 0.6 and $0.8 \mathrm{~m}$ telescopes, respectively. The two fitted lines represent their typical errors. (b) Histogram of the distribution of the rms of the differential magnitude of pairs of comparison stars. The curve is a Gaussian fit to the distribution. [See the electronic edition of the Journal for a color version of this figure.] 


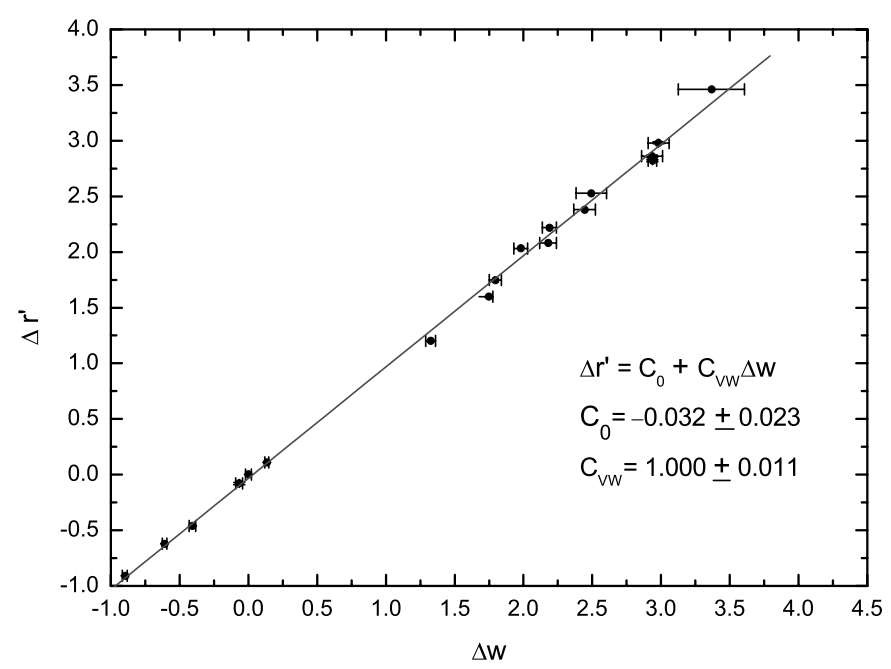

FIG. 2.- Relationship between $\Delta r^{\prime}$ and $\Delta W$ for data in a typical field. The solid line shows the fit for the data. [See the electronic edition of the Journal for a color version of this figure.]

or stars with periods out of the range, the light curve will show only noise (Vivas et al. 2004).

In practice, we distinguish subharmonics in three ways: (1) the light-curve shape, (2) narrow lines on the $\Theta$-statistic versus frequency graph relative to the widest line, which corresponds to the true frequency, and (3) reduced significance with increasing bin size (Stellingwerf 1978). We use broad bin sizes for initial scans of the full frequency range. From our experience, a subharmonic can be distinguished easily for a high $\mathrm{S} / \mathrm{N}$. But for data of a low $\mathrm{S} / \mathrm{N}$ and for a small number of observations, it can only be distinguished by the fact that the light curve of the true period looks more reasonable than that of the spurious one.

We can classify the RRLSs confirmed by us into types ab, $\mathrm{c}$, and $\mathrm{d}$. The distinction between type $\mathrm{ab}$ and type $\mathrm{c}$ is based on the light-curve shape, which is sawtoothed for type ab and sinusoidal for type c. We identify type d RRLSs to be stars with a primary-to-second period ratio of $0.74<P_{1} / P_{0}<0.75$, a physically plausible range according to stellar pulsation models (Cox et al. 1980; Kovacs et al. 1991; Bono et al. 1996). In practice, following Cseresnjes (2001), we first force the $P_{1}$ period search to be between 0.33 and 0.44 days, in order to encompass the range of primary periods of all known type d RRLSs, and fit the light curve to derive the residuals. Then we search the residuals for the second period in the range 0.45 days $<P_{0}<0.60$ days. We also repeat the above procedure by inverting the order of the period searches $\left(P_{0}\right.$ before $\left.P_{1}\right)$ in order to detect any rare double-mode RRLS (RRd) with a high amplitude in the fundamental mode relative to the first-overtone mode.

\section{RESULTS AND DISCUSSION}

In total, we took unfiltered CCD photometry of a sample of 71 candidate RRLSs that are brighter than $r^{\prime}=18.6$ out of the SDSS candidate sample (Ivezic et al. 2000). We confirmed that 69 stars are true RRLSs based on periods and light curves, including 57 type ab, 11 type c, and 1 type $d$. In our sample, only two candidate stars (004 and 122 in Ivezić et al. [2000]) do not have a reasonable light curve.

\subsection{The Catalog}

Our results are presented in a catalog of 69 RRLSs (Table 1). This table contains the star ID, right ascension and declination

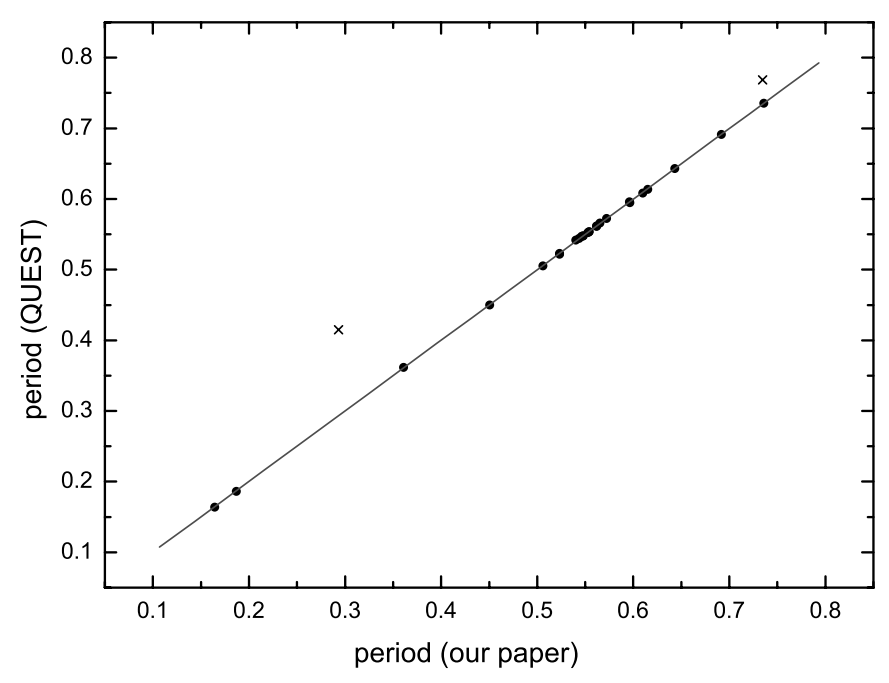

FIG. 3.-Comparison of our results with QUEST's. The solid line shows the results, fitting to $\sigma \sim 10^{-3}$. The crosses are data points with dispersion of more than $3 \sigma$. [See the electronic edition of the Journal for a color version of this figure.]

(J2000.0), number of observations for the light curve, $r^{\prime}$ amplitude, period in days, Heliocentric Julian Date at maximum light, type, and mean $r^{\prime}$ magnitude with its error. We adopt the star IDs from Ivezić et al. (2000). The symbol "q" in the last column indicates stars identified by Vivas et al. (2004).

Our catalog covers from $\alpha=10^{\mathrm{h}}$ to $15^{\mathrm{h}}$ and from $\delta=-1^{\circ} 03^{\prime}$ to $1^{\circ} 15^{\prime}$, while that of QUEST (Vivas et al. 2004) covers from $\delta=-2^{\circ} 20^{\prime}$ to $0^{\circ} 02^{\prime}$ at the same right ascension. In the $\sim 50 \mathrm{deg}^{2}$ of overlap, we measured the periods and light curves of 40 candidates, for which Vivas et al. (2001) confirmed 28 but failed to determine periods and light curves for the other 12. Vivas et al. (2004) argued that poor detection and shortage of data to calculate the periods were responsible for the unconfirmed candidates. ${ }^{3}$

Our periods agree well with those of QUEST (see Fig. 3), as indicated by the linear fit (solid line) with $\sigma \sim 7 \times 10^{-4}$, excluding stars 110 and 130 (crosses). For star 110 , our period is more reasonable because we had 47 observations compared with QUEST's 32. Moreover, our light-curve amplitude is 0.76, while that of QUEST is 0.46 . Missing phase coverage is probably responsible for these differences. We also successfully recovered star 130, which is located in Pal 5 (see $\S$ 4.2), as an RRLS. It should also be mentioned that QUEST's "alternative period" (Vivas et al. 2004) is adopted for star 77 in this figure.

\subsection{The Light Curve}

In Figure 4 we provide light curves of the 69 RRLSs folded by the PDM technique. Fourier decomposition was used to fit the light curves to measure the amplitude, time of maximum light, and mean magnitude. We also calculated the amplitudes by the equation $A^{2}=12 \sigma^{2}$ assuming a uniform distribution of data (Stellingwerf 1978), where $\sigma$ is the standard deviation of the variable RRLS magnitude (see eq. [4]). The difference in the Fourier fitting results is less than $0.06 \mathrm{mag}$, which suggests that the phase coverage of data is adequate.

Many type ab light curves in Figure 4 clearly show a bump, e.g., stars 45,115 , and 134 . The bump is always located at the

\footnotetext{
${ }^{3}$ The anonymous referee commented that eight of these stars were not detected because they were in bad columns or gaps between CCDs in the QUEST camera, and only four stars were actually observed.
} 

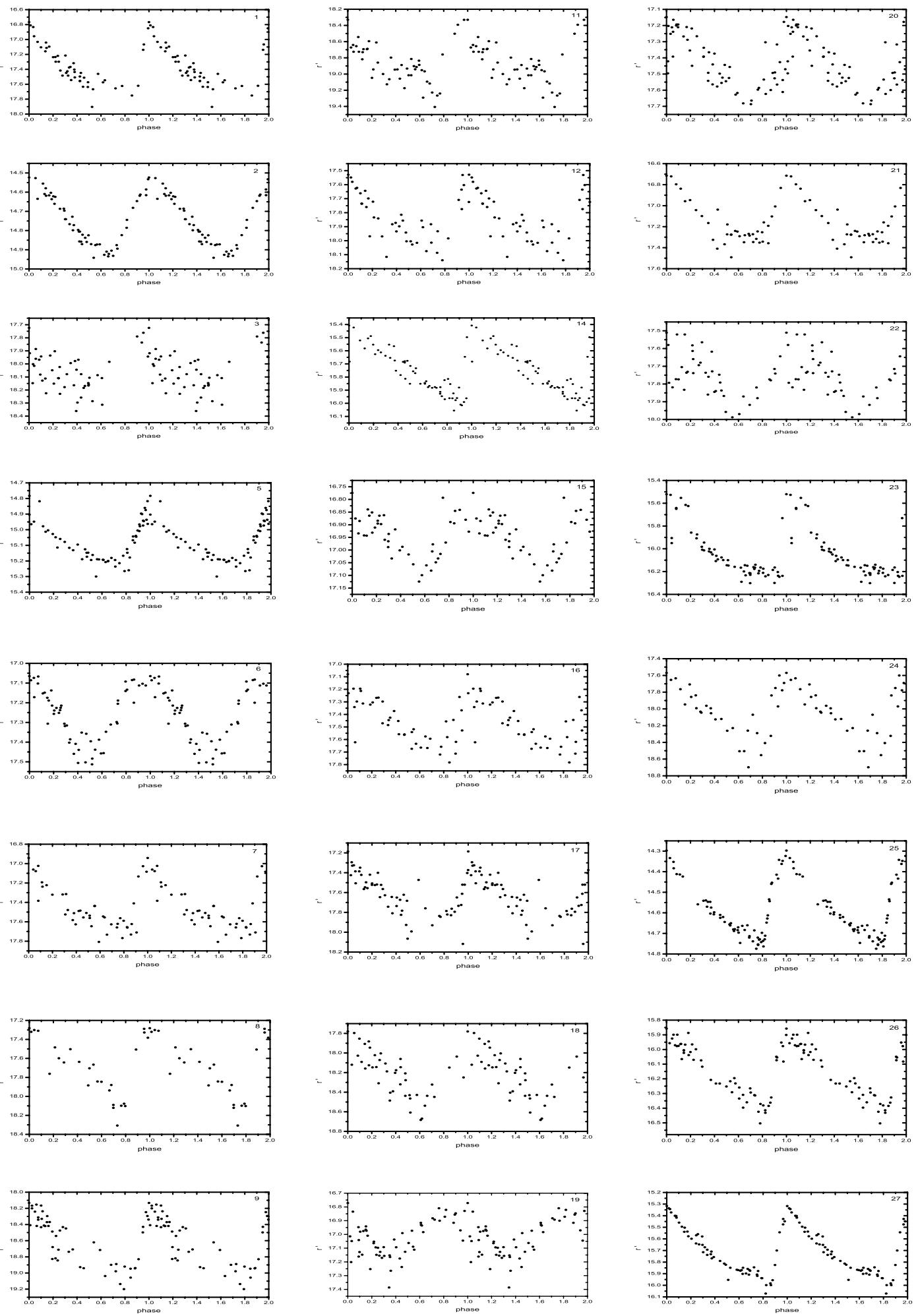

FIG. 4.- - Light curves of the RRLSs.

phase before that of minimum light. Early shock waves should be responsible for the bump according to hydrogen-line emission and line doubling from spectroscopic observations (Smith 1995). The corresponding hump that should be produced by strong main shocks, however, has not been detected in these light curves. This is because the hump, if detected, usually appears as just a slight hesitation in the increase of visible light, compared with the distinct peak followed by a minor dip near the phase before the maximum light in the UV (Smith 1995).
There are several light curves with unusually large scatter, e.g., stars 15, 48, 53, 82, 138, and 146. The scatter can result from the Blazhko effect, photometric inaccuracy, and fluctuation of comparison stars. For stars 15 and 82, the Blazhko effect may be the dominant reason because their photometric accuracy is better and their fluctuation of comparison stars smaller than average. The largest scatter for both stars exists near the maximum, which may be caused by the modulation amplitude of the Blazhko effect. For stars 48, 53, 138, and 146, the above three reasons 

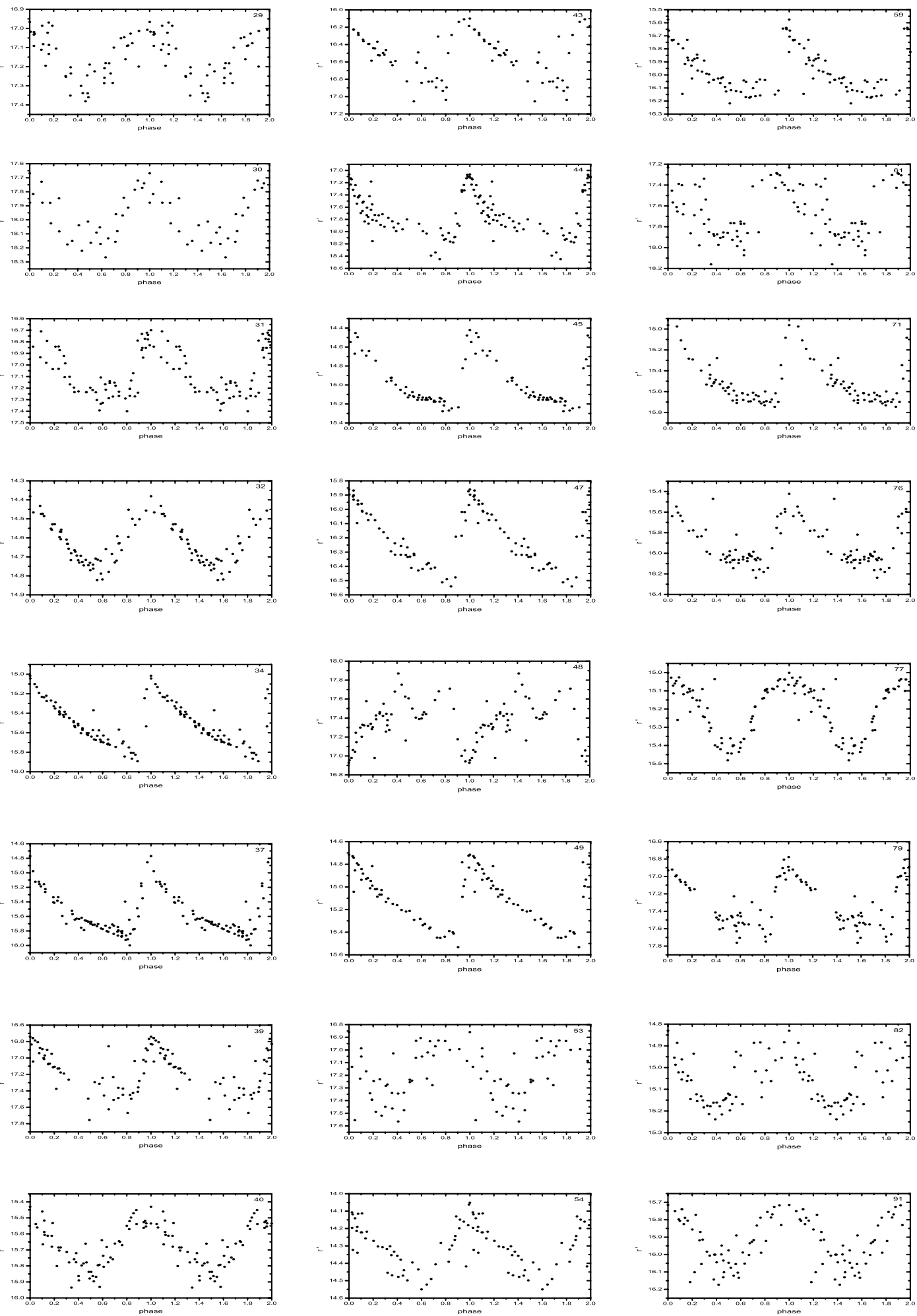

Fig. 4.-Continued

may equally contribute to the scatter and abnormal shapes of the light curves. Smith (1995) has pointed out that about $30 \%$ of known Galactic RRLSs are affected by the Blazhko effect. More observations are needed to identify the Blazhko effect and determine the modulation period by detecting more pulsational cycles.

We estimate that the contamination by other kinds of variables that have light-curve shapes and periods similar to those of RRLSs is less than $8 \%$ in our sample. Among possible contaminants, (1) W UMa eclipsing binaries should have been efficiently screened out by the selection requirement in Ivezić et al. (2000) that the candidate stars must be brighter in $r^{*}$ when they are bluer in $g^{*}-r^{*}$. (2) Anomalous Cepheids are brighter than RRLSs, with periods $\leq 2$ days and light curves resembling RRLSs. It is very difficult to recognize them in the field of the halo because it is difficult to normalize their distances for comparison with RRLSs in the color-magnitude diagram. However, according to the analysis of Vivas et al. (2004), the contamination by this kind of variable is less than $2 \%$. (3) Also, $\delta$ Scuti stars can escape the 

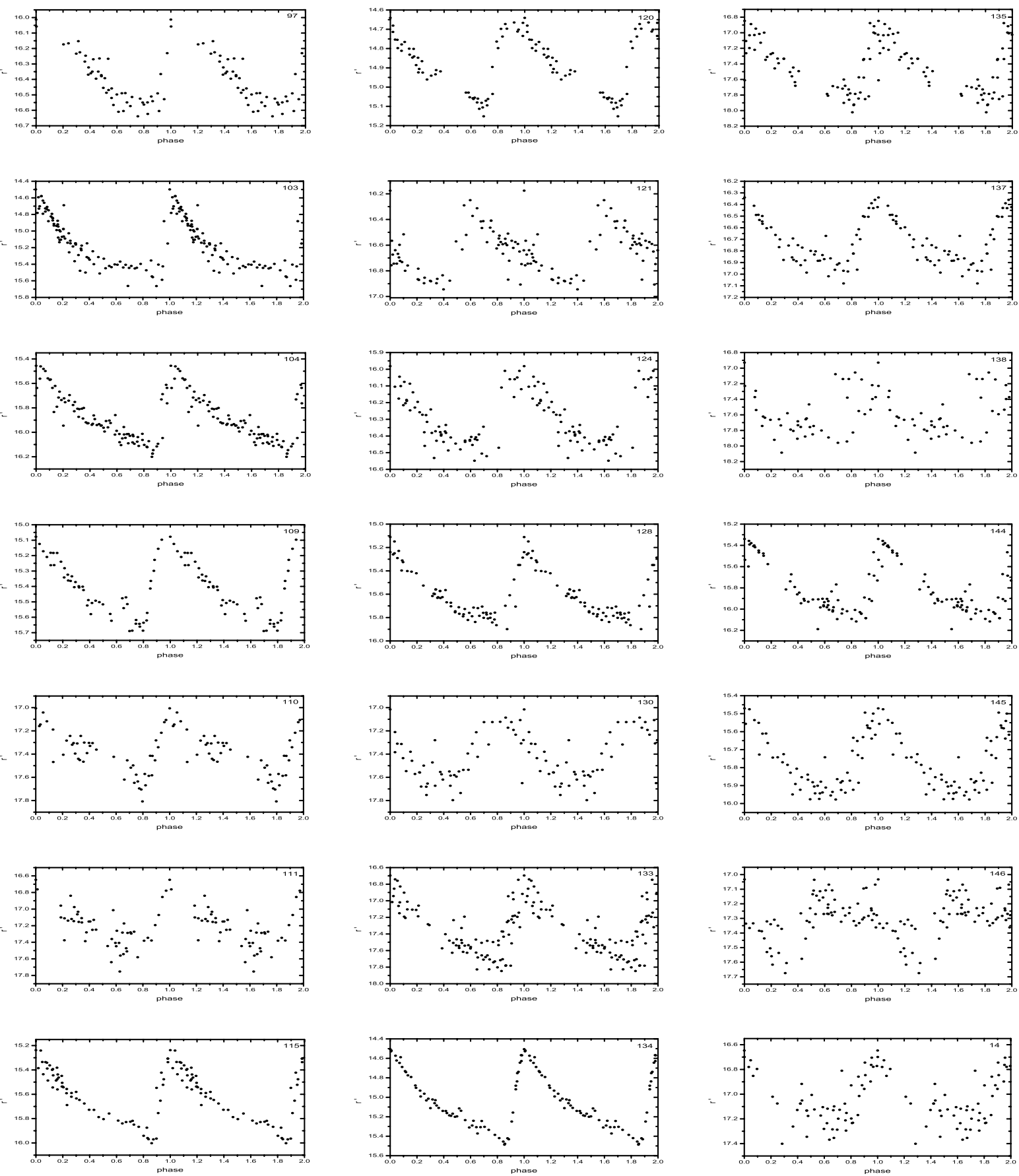

FIG. 4.-Continued

above selection constraints, since they have the same color range as RRLSs. Their light-curve shapes also look very similar to those of RRLSs because they share the same pulsation mechanism. Although $\delta$ Scuti stars are known as low-amplitude, short-period variables, high-amplitude $\delta$ Scuti stars (Rodríguez et al. 1996) exist whose amplitudes can exceed $0.1 \mathrm{mag}$ in $V$, even up to $0.5 \mathrm{mag}$ (Rodríguez et al. 1994), and whose periods can be as long as 0.2 days (Alcock et al. 2000a). In our sample, stars 29 and 77 have periods less than 0.2 days. The contamination by them is less than $3 \%$ if they are bona fide $\delta$ Scuti stars. Their pulsation parameters (Smith 1995), obtained through Fourier decomposition, are listed in Table 2 for further studies. (4) There are two stars, i.e., 004 and 122 in Ivezić et al. (2000), that do not have reasonable light curves. They contribute to the contamination by less

TABLE 2

Fourier Amplitude Ratios and Phase Differences for Stars 29 and 77

\begin{tabular}{cccccccc}
\hline \hline ID & $\begin{array}{c}\text { Period } \\
\text { (days) }\end{array}$ & $A_{1}$ & $\phi_{1}$ & $A_{21}$ & $\phi_{21}$ & $A_{31}$ & $\phi_{31}$ \\
\hline $29 \ldots \ldots \ldots . .$. & 0.164130 & 0.15 & 3.32 & 0.12 & 1.20 & 0.15 & 0.97 \\
$77 \ldots \ldots \ldots \ldots . .$. & 0.186607 & 0.19 & 3.12 & 0.18 & 6.06 & 0.02 & 3.29 \\
\hline
\end{tabular}

Notes.-The value $A_{n}$ is the ratio of the $n$th to the fundamental Fourier harmonic amplitudes, and $\phi_{n 1}$ is the normalized phase difference $\phi_{n}-n \phi_{1}$ in radians. 

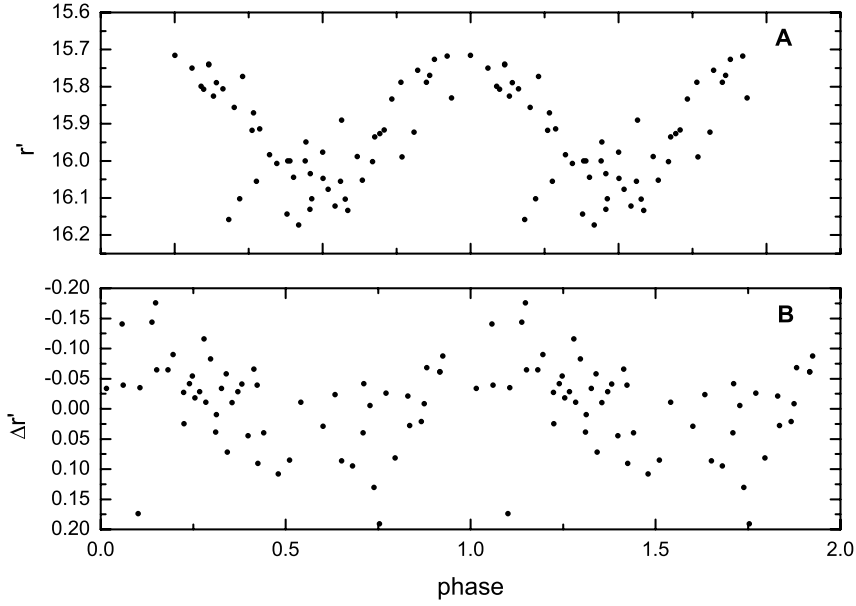

FIG. 5. - Light curves for an RRd (star 91) (a) for the fundamental mode with a period of 0.354260 days and $(b)$ for the secondary mode with a period of 0.474188 days.

than $3 \%$. In conclusion, the low contamination ratio in our sample, i.e., $8 \%$, indicates that the criteria used by Ivezić et al. (2000) to select RRLSs from the two-epoch data are very reliable.

\subsection{Double-Mode RR Lyrae Stars}

Star 91 was detected as an RRd. We show its light curve of first-overtone pulsation of the fundamental mode with a period of 0.354260 days in Figure $5 a$ and that of primary pulsation of the secondary mode with a period of 0.474188 days in Figure $5 b$.
The period ratio, $P_{1} / P_{0}$, is 0.747 , which falls into the pulsation model range from 0.741 to 0.748 (Kovacs 2001a, 2001b). The amplitude is $0.46 \mathrm{mag}$ for a fundamental-mode, first-overtone pulsation and $0.25 \mathrm{mag}$ for a secondary-mode, primary pulsation. No RRd was found by the above-mentioned "order-inverting" procedure ( $\operatorname{see} \S 3$ ), which confirms that the overtone pulsation is almost always the primary mode (Cseresnjes 2001).

RRds are very significant because they have the potential of constraining certain stellar parameters. It is well known that the pulsation periods depend on basic stellar parameters such as the mass, luminosity, effective temperature, chemical composition, and, most importantly, metallicity (Clementini et al. 2004 and references therein). These parameters can be read off a Petersen diagram (Petersen 1973), which plots the period ratio, $P_{1} / P_{0}$, against the fundamental period $P_{0}$. According to the analysis of Popielski et al. (2000), the parameters affect the position of the RRd in the Petersen diagram. They pointed out that the crucial parameter that determines the period ratio is metallicity, while the secondary ones are luminosity and mass. In addition, they argued that these parameters have a one-to-one correspondence for a given trajectory in the Petersen diagram.

We compare the position of star 91 (star) in the Petersen diagram with all known RRds identified so far in various stellar systems in Figure 6. Figure $6 a$ shows the three RRds in the Galactic bulge (asterisks), six RRds in the Galactic field (triangles), and 13 RRds in the direction of Sagittarius in the Galaxy (circles) (Mizerski 2003; Clement et al. 1993; Clementini et al. 2000; Cseresnjes 2001). Figures $6 b$ and $6 e$ show the 183 RRds in the LMC (Alcock et al. 1997, 2000b) and the 59 RRds in the SMC (Soszynski et al. 2002), respectively. Figures $6 d$ and $6 f$ show the

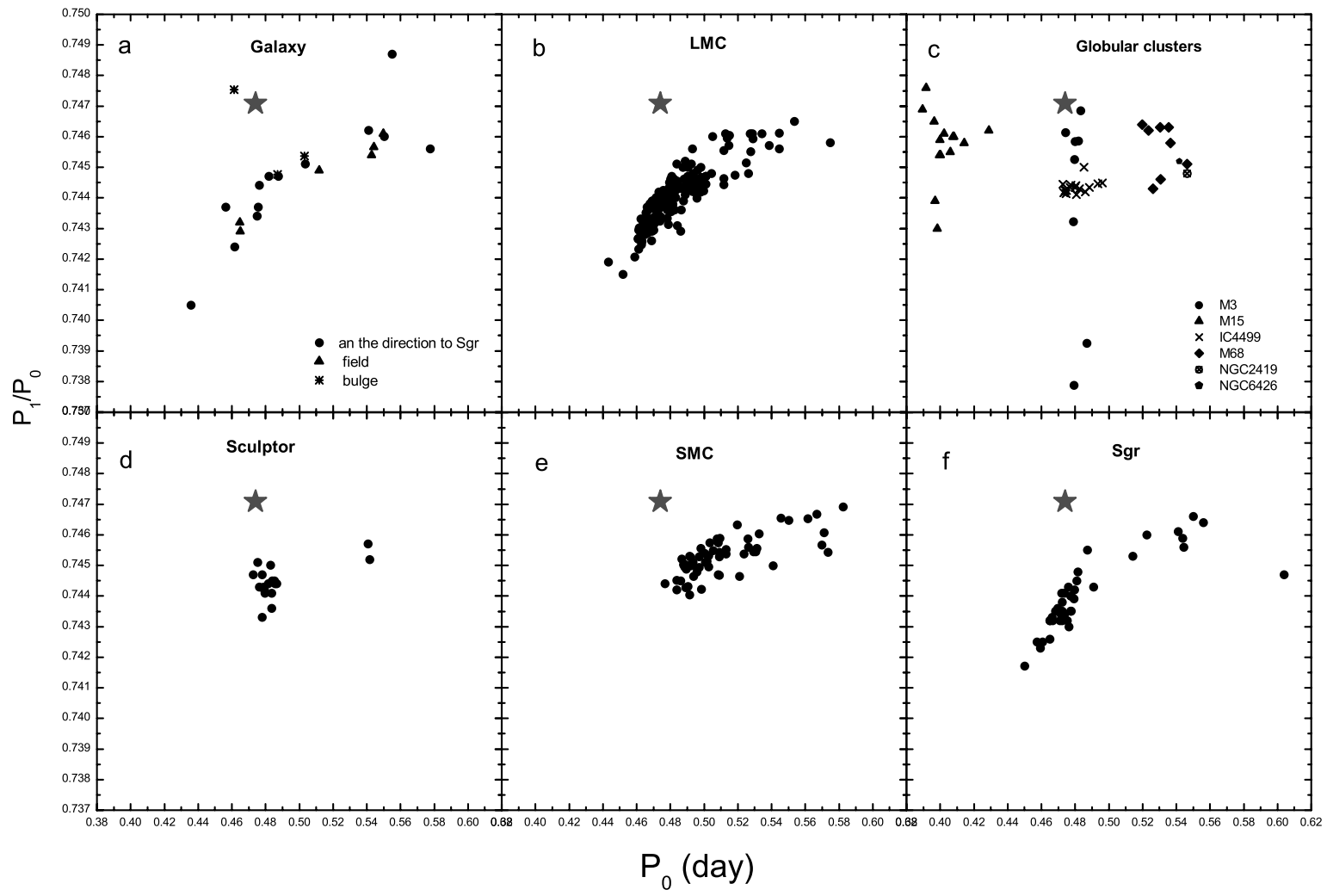

FIG. 6.-Petersen diagrams for RRds in various stellar systems. The star symbol in all panels indicates the RRd in our paper (ID 91). (a) RRds in the Galaxy (Mizerski 2003; Clement et al. 1993; Clementini et al. 2000; Cseresnjes 2001) including the bulge's, the field's, and those in the direction of Sagittarius. (b) RRds in the LMC (Alcock et al. 1997, 2000b). (c) RRds in globular clusters, i.e., M3, M15, IC 4499, M68, NGC 2419, and NGC 6426 (Clementini et al. 2004 and references therein; Clement et al. 1993; Nemec 1985; Walker \& Nemec 1996). (d) RRds in Sculptor (Kovacs 2001a). (e) RRds in the SMC (Soszynski et al. 2002). ( $f$ ) RRds in Sagittarius (Cseresnjes 2001). [See the electronic edition of the Journal for a color version of this figure.] 
RRds in two dwarf galaxies, respectively, i.e., 18 RRds in Sculptor (circles; Kovacs 2001a) and 40 RRds in Sagittarius (circles; Cseresnjes 2001). Figure $6 c$ shows the RRds in globular clusters, including eight in M3 (circles; see Clementini et al. 2004 and references therein), eight in M68 (diamonds; Clement et al. 1993), 14 in M15 (triangles; Nemec 1985), 16 in IC 4499 (crosses; Walker \& Nemec 1996), and one each in NGC 2419 (circled cross) and NGC 6426 (pentagon) (Clement et al. 1993).

Star 91 is clearly separated from the RRds in the LMC, SMC, Sagittarius, and Sculptor and from the Galactic RRds in the field and in the direction of Sagittarius in Figure 6. However, it is close to one RRd in the Galactic bulge (Fig. 6a), which is believed to have a different metallicity from the other two bulge RRds (Mizerski 2003). The star seems to reside in the distribution of the RRds in M3 in Figure $6 c$. The period ratio of the RRds in M3 has a large spread compared with those in any other stellar system, which results from a large dispersion of metallicity and/or mass (Clementini et al. 2004). Therefore, we conclude that star 91 has a large mass and/or low metallicity, based on the physical explanation of the Petersen diagram (Popielski et al. 2000), and that it is not a field star of the Galaxy. However, the star has not been reported to exist in any globular cluster. We speculate that it is a star in the tidal debris of a globular cluster either of the Galaxy or originating from a dwarf galaxy.

\subsection{The Spatial Distribution}

We calculate distances to the 69 RRLSs by assuming a constant luminosity of $M_{v}=0.56 \mathrm{mag}$ at $[\mathrm{Fe} / \mathrm{H}]=-1.6$ (Demarque et al. 2000) and transformation $M_{v}=M_{r^{\prime}}+0.44(B-V)-0.12$ (Smith et al. 2002), which typically results in $M_{v}-M_{r^{\prime}} \sim 0.04 \mathrm{mag}$. The uncertainty of the transformation is $\sim 0.1 \mathrm{mag}$, dominated by the color term $(B-V)$, which is related to the properties of horizontalbranch stars (Smith 1995). The $M_{v}$ term introduces an additional uncertainty of $\sim 0.1 \mathrm{mag}$, estimated with the Demarque et al. (2000) $M_{v}-[\mathrm{Fe} / \mathrm{H}]$ relation using $\sigma[\mathrm{Fe} / \mathrm{H}]=0.5$, which is typical of these RRLSs, and allowing for the evolutionary effect of RRLSs (see Vivas et al. 2001 and references therein). Adding in quadrature the error of the apparent $r^{\prime}$ magnitude, i.e., $\sim 0.07 \mathrm{mag}$ (see

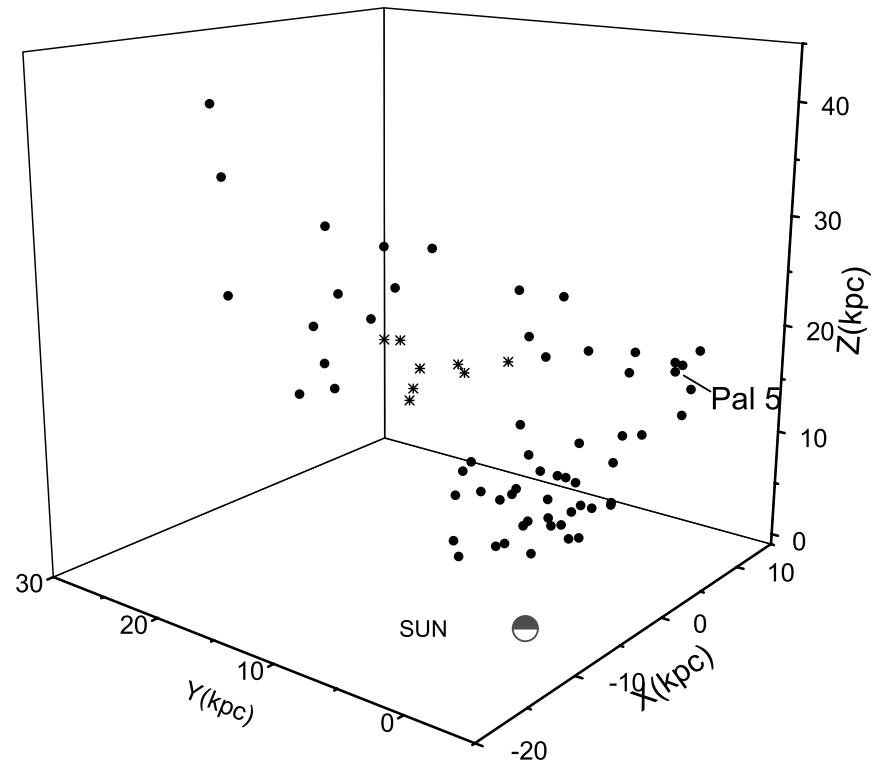

FIG. 7.-Three-dimensional view of the positions of the Sun $(X=-8,0,0)$ in kpc and the 69 RRLSs ( filled circles and asterisks) provided in Table 1 . The asterisks represent RRLSs in the $12^{\mathrm{h}} 24^{\mathrm{m}}$ clump (Zinn et al. 2004). [See the electronic edition of the Journal for a color version of this figure.]

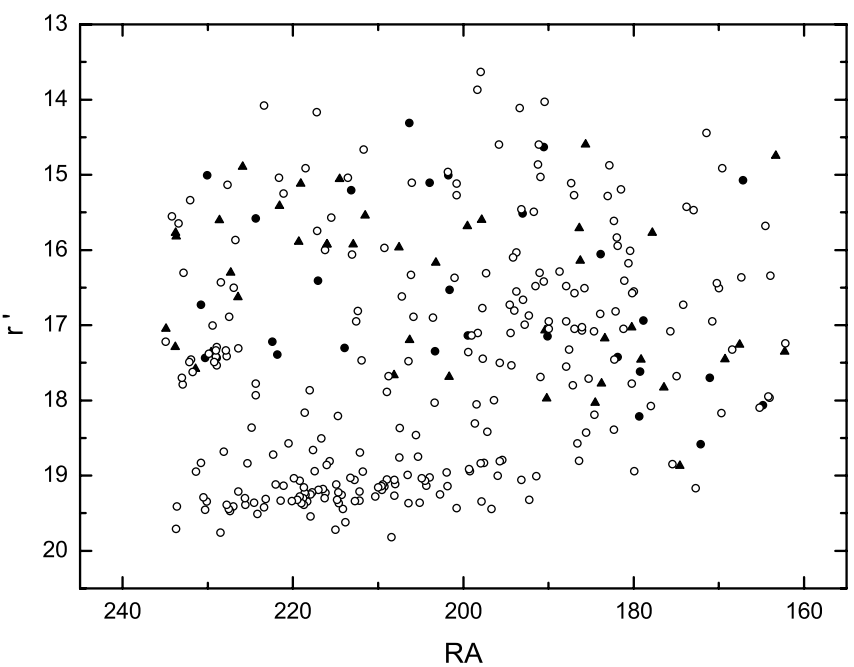

FIG. 8.-The $r^{\prime}$ magnitudes plotted against right ascension for the extended sample. Open circles show the RRLSs identified by QUEST. Triangles represent the RRLSs first confirmed in our paper. Filled circles indicate the RRLSs of QUEST recovered in our paper.

$\S 2$ ), we find that the total uncertainty of the distance modulus is $\sim 0.18 \mathrm{mag}$, corresponding to a distance precision of $8 \%$.

We plot the three-dimensional Galactic distribution of the 69 RRLSs in Figure 7, where asterisks represent the eight stars in the $12^{\mathrm{h}} 24^{\mathrm{m}}$ clump (Zinn et al. 2004). The clump spans roughly $25^{\circ}$ near $12^{\mathrm{h}} 24^{\mathrm{m}}$ and between $\sim 16.5$ and 17.5 in $V$ magnitude; it has been suggested to be part of the Sagittarius stream (Zinn et al. 2004). The eight stars of the clump in Figure 7 are centered at $X=-5.1 \mathrm{kpc}, Y=9.6 \mathrm{kpc}$, and $Z=18.9 \mathrm{kpc}$, corresponding to a distance of $22 \mathrm{kpc}$ from the Galactic center. For the six type ab RRLSs of the clump in Figure 7, we measure a mean period of 0.56 days, similar to that of the RRLSs of the clump in the catalog of Vivas et al. (2004).

We plot the mean magnitude against right ascension of the combined sample of our 69 RRLSs and the RRLSs of QUEST in Figure 8 to analyze the spatial distribution of the larger sample. In this figure, triangles represent the 41 RRLSs newly confirmed in this paper, open circles represent the RRLSs that exist only in the QUEST sample, and filled circles represent the 28 QUEST

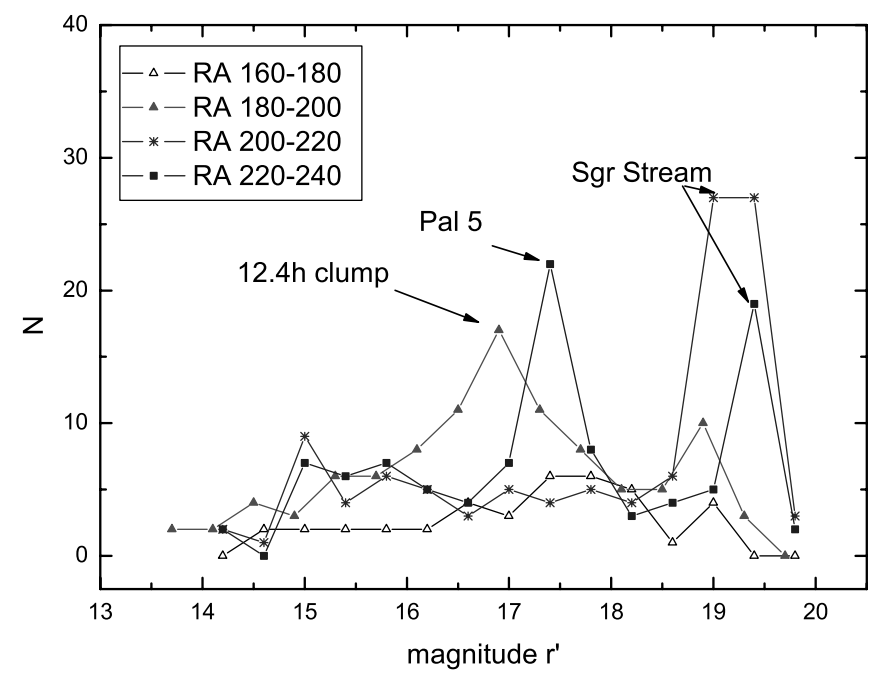

FIG. 9.-For four bands of right ascension, the number of stars $(N)$ in 0.4 mag wide bins plotted against magnitude $r^{\prime}$. [See the electronic edition of the Journal for a color version of this figure.] 
TABLE 3

RR Lyrae Stars in Pal 5

\begin{tabular}{|c|c|c|c|c|c|c|c|c|}
\hline \multicolumn{3}{|c|}{ ID } & \multirow[b]{2}{*}{$\alpha(\mathrm{J} 2000.0)$} & \multirow[b]{2}{*}{$\delta(\mathrm{J} 2000.0)$} & \multicolumn{3}{|c|}{ PERIOD (days) } & \multirow[b]{2}{*}{ TYPE } \\
\hline $\begin{array}{c}\text { Kinman \& } \\
\text { Rosino (1962) }\end{array}$ & QUEST & This Paper & & & $\begin{array}{c}\text { Kinman \& } \\
\text { Rosino (1962) }\end{array}$ & QUEST & This Paper & \\
\hline v1 & 399 & 130 & 151557 & -000652 & 0.293230 & $0.226741,0.414867^{\mathrm{a}}$ & 0.293345 & $\mathrm{c}$ \\
\hline $\mathrm{v} 2$ & 400 & $\ldots$ & 151558 & -001123 & 0.332467 & 0.332511 & $\ldots$ & $\mathrm{c}$ \\
\hline v3 & 404 & $\ldots$ & 151613 & -001003 & 0.329953 & 0.493070 & $\ldots$ & $\mathrm{c}$ \\
\hline $\mathrm{v} 4$ & 402 & $\cdots$ & 151606 & -001112 & 0.286362 & 0.401276 & $\ldots$ & $\mathrm{c}$ \\
\hline v5 & 401 & $\ldots$ & 151558 & -000547 & 0.252395 & 0.252401 & $\ldots$ & $\mathrm{c}$ \\
\hline$\cdots$ & 403 & $\ldots$ & 151609 & -001623 & $\ldots$ & 0.551669 & $\ldots$ & $\mathrm{ab}$ \\
\hline$\ldots$ & 405 & $\ldots$ & 151649 & -000746 & $\ldots$ & 0.329673 & $\ldots$ & $\mathrm{c}$ \\
\hline$\ldots$ & $\ldots$ & 133 & 151824 & +002122 & $\ldots$ & $\ldots$ & 0.529198 & $\mathrm{ab}$ \\
\hline
\end{tabular}

Note.-Units of right ascension are hours, minutes, and seconds, and units of declination are degrees, arcminutes, and arcseconds.

a Alternative period provided in the RRLS catalog (Vivas et al. 2004).

RRLSs recovered by us. To compare with the analysis of the QUEST sample by Zinn et al. (2004), we also plot a statistic graph in Figure 9 in which right ascension is divided into four bands. The clear clustering of the $12^{\mathrm{h}} 24^{\mathrm{m}}$ clump, of Pal 5, and of the Sagittarius stream is similar to what has been detected by Zinn et al. (2004). However, the small clustering at $r^{\prime} \sim 15.1$ in the $200^{\circ}-220^{\circ}$ band is more prominent than in Zinn et al. (2004). This clustering may reflect a common origin or may just be a random fluctuation in the density distribution. For further studies, we plan to do observations of metallicity and the radial velocity in the near future.

\subsection{RR Lyrae Stars in Pal 5}

We have recovered one RRLS of Pal 5, i.e., star 130. The period we measure is very consistent with that of Kinman \& Rosino (1962), who named it star V1; $\Delta P=1 \times 10^{-4}$ days. The star was also detected by QUEST (Vivas et al. 2004), but the period measured in that paper was wrong due to an insufficient number of observations. Besides, star 133 may also relate to Pal 5 because its mean $r^{\prime}$ magnitude, 17.36, is close to that of star 130 in Pal $5\left(r^{\prime}=17.43\right)$. Although located $\sim 40^{\prime}$ from the center of Pal 5, it is still within the range of the extended tails, which span $10^{\circ}$ in length and 2.5 in width and are debris originating from tidal disruption occurring in the past $100 \mathrm{Myr}$ (Odenkirchen et al. 2003). More studies are needed to tell whether the star is indeed a current member of Pal 5.

We list all eight RRLSs that have been found to relate to Pal 5 in Table 3, including five of type c identified before 2001 May (Kinman \& Rosino 1962; see also Clement et al. 2001), two RRLSs suggested by Vivas et al. (2004) that lie in the tails of $\mathrm{Pal}$ 5, and star 133 in this paper. For each star, the ID in Kinman \& Rosino (1962) is listed first, followed by that in QUEST (Vivas et al. 2004), and then by that in this paper. The fourth column is right ascension, and the fifth is declination (J2000.0). The periods measured by Kinman \& Rosino (1962), Vivas et al. (2004) in QUEST, and this paper are listed in the next three columns. The Bailey types of these RRLSs are shown in the last column.

\section{SUMMARY}

We have taken unfiltered CCD photometry of 71 candidate RRLSs in the SDSS candidate sample (Ivezić et al. 2000). Among them, 69 stars have periods and light curves typical of RRLSs. There are two candidates without a reasonable light curve. The low contamination ratio of our sample, less than $\sim 8 \%$, indicates that the criteria used by Ivezić et al. (2000) to select RRLSs from the SDSS two-epoch data are reliable.

We have presented our results in a catalog that contains the positions, periods, epochs of maximum light, and mean $r^{\prime}$ magnitudes of the 69 RRLSs. The light curve of each star is presented in Figure 4.

We have detected 1 RRd. The star shows some obvious properties of a massive star with low metallicity. It is likely an RRd in a globular cluster based on the Petersen diagram. We have analyzed the spatial distribution of the combined sample of our 69 RRLSs and the QUEST sample and compared it with the QUEST results of Zinn et al. (2004). We have also collected the data of all RRLSs related to Pal 5 and added a new RRLS to the cluster. Our measurements provide valuable probes of the shape and origin of the Galactic halo.

Observations of radial velocities and metal abundances are required for further studies of RRLSs in order to investigate the origin of the Galactic stellar halo. We have proposed measuring the metal abundances of the RRLSs of this sample in the next year.

We are grateful to the anonymous referee for valuable comments. We acknowledge S. Y. Jiang, Z. P. Li, Y. X. Zhang, and W. H. Bian for many useful suggestions. We would like to thank all the staff at the Xinglong station of the NAOC, especially J. Y. Wei, X. J. Jiang, and X. M. Lu for their assistance during the observation. We also thank Y. Shen, J. Z. Li, and the night assistants for their patient company through the long nights. Y. Q. Lou is greatly acknowledged for granting us observation time with the TNT. We also want to acknowledge A. K. Vivas for providing the catalog of RRLSs by e-mail.
Alcock, C., et al. 1997, ApJ, 482, 89

.2000a, ApJ, 536, 798

2000b, ApJ, 542, 257

Baade, W. 1944, ApJ, 100, 147

Bono, G. 2003, in Stellar Candles for the Extragalactic Distance Scale, ed. D. Alloin \& W. Gieren (Berlin: Springer), 85

\section{REFERENCES}

Bono, G., Filippina, C., Vittorio, C., \& Marcella, M. 1996, ApJ, 471, L33

Bullock, J. S., Kravtsov, A. V., \& Weinberg, D. H. 2000, ApJ, 539, 517 2001, ApJ, 548, 33

Chiba, M., \& Beers, T. C. 2001, ApJ, 549, 325

Clement, C. M., Ferance, S., \& Simon, N. R. 1993, ApJ, 412, 183

Clement, C. M., et al. 2001, AJ, 122, 2587 
Clementini, G., Corwin, T. M., Carney, B. W., \& Sumerel, A. N. 2004, AJ, 127, 938

Clementini, G., et al. 2000, AJ, 120, 2054

Cox, A. N., Hodson, S. W., \& King, D. S. 1980, ApJ, 236, 219

Cseresnjes, P. 2001, A\&A, 375, 909

Demarque, P., Zinn, R., Lee, Y., \& Yi, S. 2000, AJ, 119, 1398

Eggen, O. J., Lynden-Bell, D., \& Sandage, A. R. 1962, ApJ, 136, 748

Gould, A., Guhathakurta, P., Richstone, D., \& Flynn, C. 1992, ApJ, 388, 345

Ibata, R., Lewis, G. F., Irwin, M., Totten, E., \& Quinn, T. 2001, ApJ, 551, 294

Irwin, M., \& Hatzidimitriou, D. 1995, MNRAS, 277, 1354

Ivezić, Ž., et al. 2000, AJ, 120, 963

Kinman, T. D. 2002, Inf. Bull. Variable Stars, 5354, 1

Kinman, T. D., \& Rosino, L. 1962, PASP, 74, 499

Kong, M.-Z., Qiu, Y.-L., Shen, C.-J., Deng, J.-S., Zhang, B., \& Hu, J.-Y. 2002, Chinese J. Astron. Astrophys., 2, 463

Kovacs, G. 2001a, A\&A, 375, 469

- 2001b, in Stellar Pulsation-Nonlinear Studies, ed. M. Takeuti \& D. D. Sasselov (Dordrecht: Kluwer), 61

Kovacs, G., Buchler, J. R., \& Marom, A. 1991, A\&A, 252, L27

Kuhn, J. R., Smith, H. A., \& Hawley, S. L. 1996, ApJ, 469, L93

Lafler, J., \& Kinman, T. D. 1965, ApJS, 11, 216

Layden, A. C. 1997, PASP, 109, 524

Majewski, S. R. 1993, ARA\&A, 31, 575

Majewski, S. R., Ostheimer, J. C., Patterson, R. J., Kunkel, W. E., Johnston, K. V., \& Geisler, D. 2000, AJ, 119, 760

Majewski, S. R., Skrutskie, M. F., Weinberg, M. D., \& Ostheimer, J. C. 2003, ApJ, 599, 1082

Mizerski, T. 2003, Acta Astron., 53, 307

Nemec, J. M. 1985, AJ, 90, 240

Newberg, H. J., et al. 2002, ApJ, 569, 245

Norris, J. E. 1994, ApJ, 431, 645

Odenkirchen, M., et al. 2003, AJ, 126, 2385
Petersen, J. O. 1973, A\&A, 27, 89

Popielski, B. L., Dziembowski, W. A., \& Cassisi, S. 2000, Acta Astron., 50, 491

Riess, A. G., et al. 1999, AJ, 118, 2675

Rodríguez, E., López de Coca, P., Rolland, A., Garrido, R., \& Costa, V. 1994, A\&AS, 106, 21

Rodríguez, E., Rolland, A., López de Coca, P., \& Martín, S. 1996, A\&A, 307, 539

Searle, L. 1977, in The Evolution of Galaxies and Stellar Populations, ed. B. M.

Tinsley \& R. B. Larson (New Haven: Yale Univ. Press), 219

Searle, L., \& Zinn, R. 1978, ApJ, 225, 357

Smith, H. A. 1995, RR Lyrae Stars (Cambridge: Cambridge Univ. Press)

Smith, J. A., et al. 2002, AJ, 123, 2121

Soszynski, I., et al. 2002, Acta Astron., 52, 369

Stellingwerf, R. F. 1978, ApJ, 224, 953

Vivas, A. K., \& Zinn, R. 2003, Mem. Soc. Astron. Italiana, 74, 928

Vivas, A. K., Zinn, R., \& Gallart, C. 2005, AJ, 129, 189

Vivas, A. K., et al. 2001, ApJ, 554, L33 2004, AJ, 127, 1158

Walker, A. R., \& Nemec, J. M. 1996, AJ, 112, 2026

Wyse, R. F. G. 1999a, in ASP Conf. Ser. 165, The Third Stromlo Symp.: The Galactic Halo, ed. B. K. Gibson, T. S. Axelrod, \& M. E. Putman (San Francisco: ASP), 1

- 1999b, in The Formation of Galactic Bulges, ed. C. M. Carollo, H. C. Ferguson, \& R. F. G. Wyse (Cambridge: Cambridge Univ. Press), 195

Yanny, B., et al. 2000, ApJ, 540, 825 2003, ApJ, 588, 824

Zinn, R. 1993, in ASP Conf. Ser. 48, The Globular Cluster-Galaxy Connection, ed. G. H. Smith \& J. P. Brodie (San Francisco: ASP), 38

Zinn, R., Vivas, A. K., Gallart, C., \& Winnick, R. 2004, in ASP Conf. Ser. 327, Satellites and Tidal Streams, ed. F. Prada, D. Martinez-Delgado, \& T. J. Mahoney (San Francisco: ASP), 92 